\title{
Additional challenges to fair representation in autistic advocacy
}

McCoy at el make an important contribution to a difficult topic. Both historically and today, people with psychiatric diagnoses have often been treated in harmful ways. Advocates who have been diagnosed have fought back against this. McCoy et al outline useful strategies to help ensure that advocacy by autistic individuals represents a range of views. They outline a notion of fair representation whereby non-elected advocates can successfully represent the views of an entire population (in this case all autistic people). They contrast this with partial representation whereby non-elected advocates believe they speak for the entire population but actually only represent the views of part of that population. I do not object to any of McCoy et al's specific suggestions for achieving fair representation in autistic advocacy since they all appear to be good ideas. Rather, I here outline some challenges for achieving fair representation in autism advocacy which McCoy et al do not discuss. In doing so I draw on my own experience of being autistic and of interacting with autistic advocates both face-to-face and online.

McCoy et al outline two different types of autism advocacy. On the one hand, they discuss autistic individuals who see an autistic way of being as equally legitimate to any other way of being and feel that the problems that autistic people often currently face lie in how society is set up (the 'neurodiversity' view). On the other hand, there are parents and carers of autistic individuals who feel that autism is a debilitating disability and seek significant support for their relatives. Much of McCoy et al's paper focuses on the divide between these two groups. However, there are other important views amongst autistic advocates which McCoy et al do not adequately discuss. There are some autistic neurodiversity advocates who believe that although some forms of autism give rise to alternative ways of being that are as good as any other, autism can manifest in a wide variety of ways and some of these can be highly impairing. Those who adopt such a viewpoint pose no particular challenge for McCoy et al's argument. In contrast some autistic advocates are very anti-neurodiversity and these do pose a significant challenge for practically implementing McCoy et al's approach. Anti-neurodiversity autistic advocates generally take autism to be an intrinsic disability rather than an alternative way of being and they do not see society as a major source of the problems which autistic people face (they would reject the social model of disability). Those with such views often feel that the neurodiversity movement is misrepresenting what it is like to be autistic. McCoy et al do mention that some autistic people reject the claims of the neurodiversity movement, but they do not discuss this position (and the problems it poses for 'fair representation') in any detail. Based on my own experience I suggest that the portion of autistic individuals who oppose the claims of the neurodiversity movement is higher than McCoy et al's paper might suggest. A more significant problem for McCoy et al's argument is that there is a great deal of hostility and division between pro-neurodiversity and anti-neurodiversity autistic individuals. Both sides seem to think that the other side is harming autistic people, and sometimes even disparage the motives of their opponents. I suspect that the levels of hostility in such debates means that many autistic advocates, both those who are pro-neurodiversity and those who are anti-neurodiversity, would 
feel little obligation to promote the views of their opponents. However, willingness to fairly portray both sides of the debate seems to be a prerequisite for meeting notions of fair representation.

A second challenge to achieving fair representation may come from a particular characteristic associated with autism. Autistic individuals can have a tendency to struggle to see the point of view of others. They can have a very focused interest in and understanding of their own views whilst not placing their own views within a wider context. This means that they can struggle to shift their focus and understanding from their own views to the views of others. I am aware that I do this and I actively take steps to mitigate it but I feel I usually only have limited success at seeing the views of others. To the degree to which any particular autistic person struggles to see the views of others there will be a significant challenge to successful fair representation. Additionally, it seems possible that difficulties in seeing the perspective of others might partially explain why there is so much hostility between pro-neurodiversity and antineurodiversity advocates.

A third challenge comes from problems associated with online interaction. As McCoy at el note, autistic people often prefer interacting online compared to face-to-face interactions. However, there are well known problems associated with online debate. There is the problem of the echo chamber whereby discussion becomes a mutually self-supporting confirmation of views. There is also the problem of polarization whereby discussions between individuals with opposing views can primarily consist of short, hostile exchanges on social media or forums rather than sustained back and forth discussion where both sides properly listen to the other side. These are problems of online discussion in general rather than being restricted to autism advocacy. However, to the extent that any particular autistic person tends to avoid face-to-face communication and spends extra time online, such issues could have a greater than usual influence.

Finally, McCoy et al briefly mention an alternative approach which might mitigate some challenges to fair representation. They outline how populations can be subdivided into smaller subgroups. This means that a member of that smaller subgroup can aim to only represent that smaller subgroup rather than claim that they represent the entire population. This potentially makes it easier to ensure that any particular individual does fairly represent those they advocate for (whilst also being able to come together with advocates of other subgroups to advocate for the entire population). McCoy et al mention how the autistic spectrum had, until the 2013 DSM5 , a number of subtypes. They briefly mention that subtypes of autism might form the basis for smaller subgroups which could be the basis for advocacy whereby members of a subtype primarily only advocate on behalf of autistic individuals with that subtype.

I think that adding substantive subtypes to the autistic spectrum could significantly help autistic advocacy (for further discussion see Fellowes 2017). Having myself attempted autistic advocacy, including been consulted in the capacity of an autistic individual on a scientific project researching autism, I have tried to ensure I described a broader perspective than just my own experiences. However, I think this is a challenging task. Simply knowing that autism can 
manifest in a large variety of ways does not then specify the ways in which it manifests. In contrast, if autism had a number of substantive subtypes then I would have greater guidance over the ways in which autism can manifest. This would help increase my awareness that autism can manifest in ways other than my own and, if I did advocate for the wider autistic spectrum, would help me represent a variety of ways in which autism can manifest.

\section{Bibliography}

Fellowes, S. 2017. Putting the present in the history of autism. Studies in the History and Philosophy of the Biological and Biomedical Sciences 61:54-58. 